\title{
Variação na qualidade do azeite em cultivares de oliveira
}

\author{
Luiz Fernando de Oliveira da Silva ( $\left.{ }^{1}\right)$; Adelson Francisco de Oliveira ( $\left.{ }^{2}\right)$; Rafael Pio ( $\left.{ }^{1 *}\right)$; Tatielle \\ Custódio Alves ( ${ }^{2}$ ); Carolina Ruiz Zambon $\left({ }^{2}\right)$ \\ (') Universidade Federal de Lavras - UFLA, Dep. de Agricultura, Caixa Postal 3037, 37200-000 Lavras (MG), Brasil. \\ (2) Empresa de Pesquisa Agropecuária de Minas Gerais - EPAMIG-CTSM, Caixa Postal 176, 37200-000 Lavras (MG), Brasil. \\ (*) Autor correspondente: rafaelpio@dag.ufla.br
}

Recebido: 10/fev./2012; Aceito: 5/jun./2012

\section{Resumo}

O cultivo de oliveira (Olea europaea L.) no sul de Minas Gerais tem apresentado resultados promissores quanto à produção de frutos. No entanto, há necessidade de verificar seu valor nutritivo e a qualidade do azeite oriundo dos frutos das cultivares potencialmente produtivas. Objetivou-se com este trabalho determinar a composição centesimal dos frutos, as características químicas e o perfil de ácidos graxos dos azeites extraídos de diferentes cultivares de oliveira, com a finalidade de selecionar os materiais com potencial agroindustrial. Foram avaliadas 32 cultivares de oliveira com seis anos de idades quanto à composição centesimal (teores de umidade, lipídeos, proteínas e cinzas, tanto na polpa quanto no caroço dos frutos); composição química e perfil de ácidos graxos dos azeites. Observaram-se nas cultivares MGS ASC315, Cerignola, MGS GRAP561 e MGS GRAP575 maiores valores de lipídeos. Além disso, as análises indicaram que o caroço possui quantidades significativas de lipídeos, embora sempre inferiores às quantidades presentes na polpa. Os índices de acidez em ácido oleico, peróxidos e refração absoluta estão de acordo com a resolução da ANVISA, podendo ser inicialmente classificados como azeite de oliva extravirgem ou virgem. De modo geral, todos os ácidos graxos presentes nos azeites possuem valores satisfatórios, destacando a cultivar MGS GRAP084, com maior concentração de ácido oleico, principal ácido responsável pelos benefícios à saúde.

Palavras-chave: Olea europaea L., acidez, ácidos graxos, ácido oleico.

\section{Quality variation of olive oil in olive cultivars}

\begin{abstract}
Olive tree (Olea europaea L.) grown in south Minas Gerais State has shown promising results for fruit yield. However, little is still known about the fruit nutritional value and oil quality for those potentially productive cultivars. The objective of this work was to determine fruit centesimal composition, chemical characteristics, and the fatty acids profile of oils extracted from different olive tree cultivars to select the ones with improved agro-industrial potential. We assessed 32 six-year-old olive cultivars for the centesimal composition (moisture, lipids, proteins, and ash content, both in flesh and stone fruits), chemical composition, and fatty acid profiles. The cultivars MGS ACS315, Cerignola, MGS GRAP561, and MGS GRAP575 showed higher lipid content. Besides, these results indicated that the fruit core showed significant lipid amounts, although lower than those present in the pulp. The index results of oleic acid, peroxides, and absolute refraction are in agreement with ANVISA resolution and initially may be classified as virgin or extra virgin oil. In general, all fatty acids present in the olive oil tested had satisfactory values and the MGS GRAP084 cultivar had the highest content of oleic acid, the main fatty acid responsible for health benefits
\end{abstract}

Key words: Olea europaea L., acidity, fatty acid, oleic acid.

\section{INTRODUÇÃO}

Na América do Sul, a Argentina e o Chile são os principais produtores e exportadores de azeitona e azeite, respectivamente, com 100 mil e 10 mil hectares plantados.

O Brasil é considerado um dos maiores importadores mundiais de azeitonas e derivados. Em 2009, foram importadas, aproximadamente, 44 mil toneladas de azeite e 70 mil toneladas de azeitonas em conservas, movimentando mais de um bilhão de reais, no mercado nacional, com esses produtos (Oliveira et al., 2012b).
Nos últimos anos, iniciaram-se trabalhos em Minas Gerais e no Rio Grande do Sul com o intuito de viabilizar a expansáo da olivicultura nacional. Os resultados foram animadores, do ponto de vista propagativo e fitotécnico, em que se pode viabilizar a propagação da oliveira por estaquia (Oliveira et al., 2010b; 2012a) e, ainda, se recomendar informaçóes técnicas visando à produção de frutos (Vieira Neto et al., 2008). Porém, a olivicultura, principalmente em Minas Gerais, náo se tornou uma alternativa rentável e viável para os produtores, devido à falta de conhecimento das características agroindustriais 
das cultivares introduzidas e sua potencialidade econômica de extração e comercialização do azeite produzido. Entre essas características, a composição centesimal dos frutos e os parâmetros químicos se destacam para o conhecimento, a seleção e recomendação de cultivares superiores.

Atualmente, na região da Serra da Mantiqueira existem cerca de 300 mil oliveiras plantadas. A cultivar Arbequina corresponde a cerca de 50\% dessa quantidade, seguida das cultivares MGS GRAP541, MGS Mariense e Koroneiki, todas destinadas à produção de azeite.

Para que o azeite seja comercializado no Brasil, precisa estar dentro dos padróes exigidos pela ANVISA, sendo o azeite extravirgem o produto extraído do fruto da oliveira unicamente por processos mecânicos ou outros meios físicos, sob controle de temperatura adequa$\mathrm{da}$, mantendo-se a natureza original do produto e observados os valores dos parâmetros de qualidade com base em análises físico-químicas que o qualificarão (AuEdPimentel et al., 2008, MAPA, 2012).

Alguns pesquisadores realizaram a caracterização fisico-química de algumas amostras de azeite extraídas no sul de Minas Gerais. CArdoso et al. (2010) analisaram cinco amostras de azeite e Oliveira et al. (2010a), seis amostras de azeites produzidos no sul de Minas Gerais, sendo verificadas características positivas em todas as amostras.

Diante do exposto, o objetivo deste trabalho foi determinar a composição centesimal dos frutos, índices de qualidade e perfil de ácidos graxos dos azeites extraídos de diferentes cultivares de oliveira na Região Sul de Minas Gerais, com a finalidade de selecionar aquelas que possuem qualidade e potencial agroindustrial de comercialização.

\section{MATERIAL E MÉTODOS}

As oliveiras utilizadas neste trabalho pertencem ao Banco Ativo de Germoplasma (BAG), situado na Fazenda Experimental da EPAMIG, em Maria da Fé, sul de Minas Gerais, a $22^{\circ} 18^{\prime}$ de latitude Sul e $45^{\circ} 23^{\prime}$ de longitude Oeste, altitude média de 1.276 metros e classificação climática de Köppen tipo $\mathrm{Cwb}$, ou seja, clima temperado chuvoso (mesotérmico), também denominado subtropical de altitude (Figura 1) e solo do tipo Latossolo Vermelho e textura média argilosa.

O pomar foi instalado em 2005, com plantas obtidas a partir de estacas enraizadas, segundo as recomendaçóes de Pio et al. (2005), sendo seis plantas para cada cultivar. Foram coletados frutos de 32 cultivares de oliveira: Alto D'Ouro, Arbequina, Ascolano USA, Cerignola, Empeltre, Galega, JB 1, MGS ASC315, MGS ASC322, MGS ASC323, MGS GRAP084, MGS GRAP541, MGS GRAP550, MGS GRAP553, MGS GRAP556, MGS GRAP561, MGS GRAP575, MGS MANZ215, MGS MANZ234, MGS MARIENSE, MGS MISS293, MGS NEBLINA, MGS ROP398, MGS SAL488,
MGS SEVERO, MGS TAF390, MGS TAF391, MGS VIGLIONE, MGS ZAL010, Mission, Negroa e Picual.

\section{Composição centesimal}

As azeitonas de cada cultivar foram lavadas em água corrente e separadas em polpa e caroço para realização das análises. O delineamento experimental foi o inteiramente casualizado (DIC), em três repetiçóes, sendo utilizado, inicialmente, $300 \mathrm{~g}$ de frutos por parcela. As análises foram realizadas de acordo com as instruçóes estabelecidas pela Association of Official Analytical Chemists (AOAC, 2005).

Para a determinação da umidade, foi empregada a técnica gravimétrica, que corresponde à perda de massa quando um produto é aquecido. A amostra fracionada foi pesada em cápsulas de porcelana, procedendo-se, em seguida, à secagem em estufa a $65^{\circ} \mathrm{C}$, até a obtenção de massa constante.

Para a determinação de lipídeos foram trituradas $3 \mathrm{~g}$ da amostra seca em aparelho Tecnal ${ }^{\circledR}$. O método utilizado consiste em se fazer a extração contínua em aparelho tipo "soxhlet" por meio de determinador de gordura TE 044 Tecnal", utilizando-se éter como solvente.

A análise da proteína bruta foi realizada por meio de digestáo. $\mathrm{O}$ procedimento passou por três etapas: a primeira, digestão da matéria orgânica, partindo-se de uma amostra de $1 \mathrm{mg}$ de massa seca desengordurada com solução de $\mathrm{H}_{2} \mathrm{SO}_{4}+\mathrm{CuSO}_{4}$; a segunda, destilação em determinador de nitrogênio Tecnal ; e a terceira etapa, a titulação com $\mathrm{HCl} 0,02 \mathrm{~N}$.

Foram pesados em cadinhos $1 \mathrm{~g}$ de massa seca desengordurada de cada cultivar. A incineração foi feita em bico de gás, aquecendo-se igualmente todas as faces do cadinho. Após a completa carbonização do material, fez-se a transferência do cadinho para a mufla aquecida a $550{ }^{\circ} \mathrm{C}$, onde permaneceu por aproximadamente 6 horas. Os cadinhos foram retirados da mufla e colocados em dessecador até o resfriamento e, em seguida pesados.

Os resultados foram submetidos à análise de variância e as médias comparadas pelo teste de Scott-Knott a 5\% de probabilidade, através do software estatístico Sisvar (FERreira, 2011).

\section{Composição química e perfil de ácidos graxos}

As análises químicas dos azeites foram realizadas utilizando o sistema Abencor (SuÁrez et al., 1975) adaptado para que se obtivessem quantidades de azeites suficientes para a realização das análises.

Os frutos de cada cultivar foram lavados em água corrente, sendo retiradas as impurezas, como folhas e detritos, para posterior trituração em moinho metálico. 


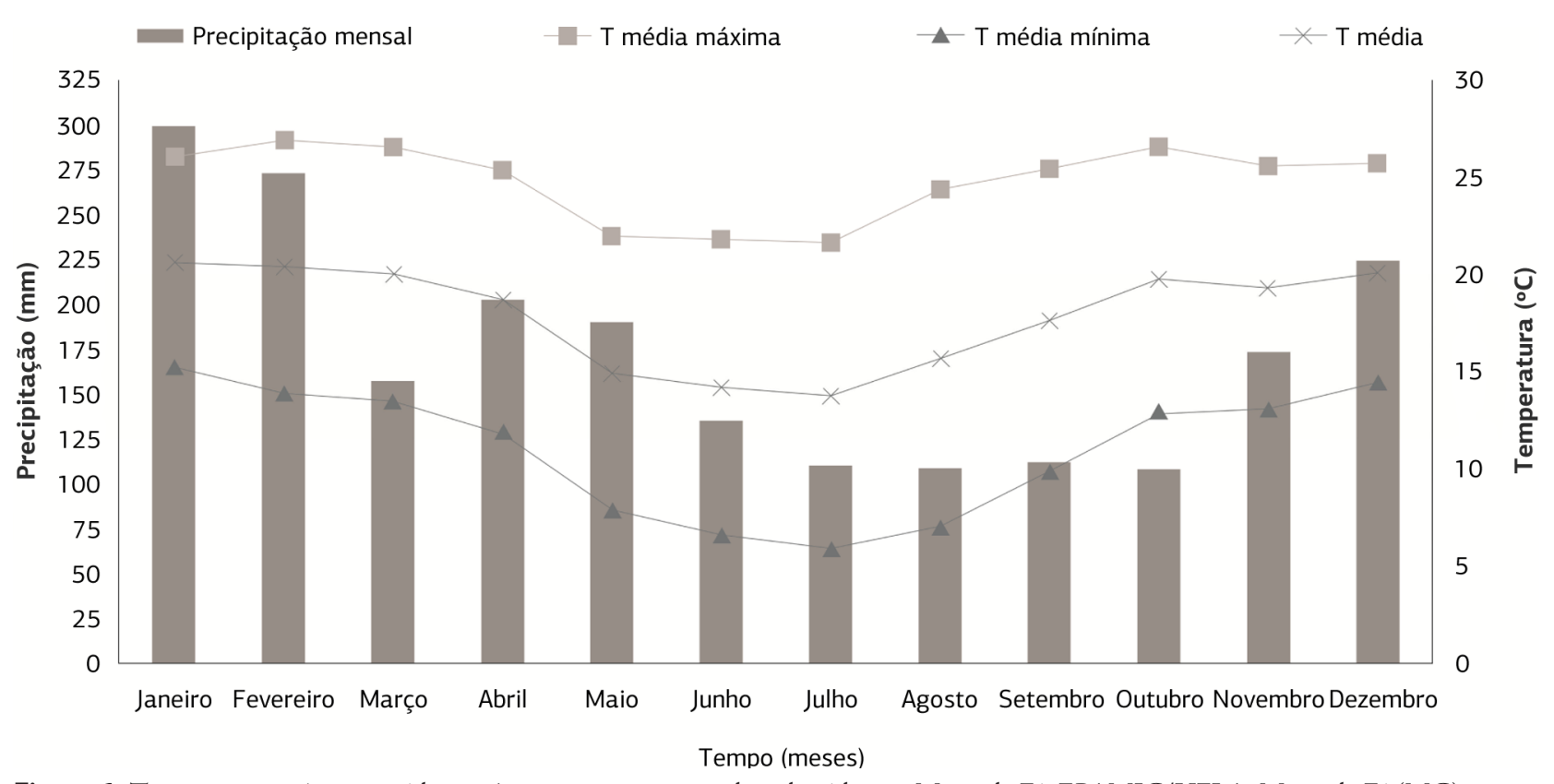

Figura 1. Temperatura máxima, média e mínima e precipitação pluvial média em Maria da Fé. EPAMIG/UFLA, Maria da Fé (MG), 2011.

A amostra foi aquecida a $28^{\circ} \mathrm{C}$ (SÁnCHEs et al., 2005) durante o processo de homogeneização realizado por uma batedeira convencional de uso culinário, com dois tipos de movimento, translação e rotação das pás, durante um intervalo de 50 a 60 minutos.

Com o uso de uma balança analítica, foram separadas $450 \mathrm{~g}$ da amostra e levadas até a centrífuga de alta rotaçáo, com $4.200 \mathrm{rpm}$ e durante dois minutos, para possibilitar a separação do azeite.

O azeite foi acondicionado em copos plásticos, onde permaneceu por 60 minutos para a decantaçáo de possíveis resíduos. Após esse processo, o azeite foi transferido para recipiente de vidro âmbar, com o auxílio de uma seringa.

As amostras de azeites foram analisadas no laboratório da empresa Oli'Ma Indústria de Alimentos Ltda ${ }^{\circledR}$, onde foram realizadas as seguintes análises em triplicata, de acordo com as normas do Instituto Adolfo Lutz (IAL, 2005) e da Association of Official Analytical Chemists (AOAC, 2005):

- Acidez em ácido oleico: determinado por titulação com solução de éter etílico, álcool e indicador fenolftaleína e os resultados expressos em percentagem de ácido oleico.

- Índice de peróxidos: determinado pela capacidade da amostra em oxidar iodeto de potássio e os resultados expressos em miliequivalentes ou $\mathrm{mmol}_{\mathrm{c}} \mathrm{kg}^{-1}$.

- Índice de iodo: determinado pelo método de Wijs, o qual é aplicável a todos os óleos e gorduras normais que não contenham ligaçóes duplas conjugadas. $\mathrm{O}$ índice de iodo de um óleo ou gordura é a medida do grau de insaturação e é expresso em termos do número de centigramas de iodo absorvido por grama da amostra (\% iodo absorvido).

- Índice de refração absoluta: determinado através de refratômetro de Abbe, realizando-se a leitura na escala que resulta diretamente no índice de refração absoluto a $40{ }^{\circ} \mathrm{C}$. O índice de refração é característico para cada tipo de óleo, dentro de certos limites. Está relacionado com o grau de saturação das ligaçôes, mas é afetado por outros fatores tais como: teor de ácidos graxos livres, oxidação e tratamento térmico.

- Absorbância em ultravioleta: determinada por exame espectrofotométrico das amostras na regiāo do ultravioleta. A absorção em 270 e $232 \mathrm{~nm}$, comprimentos de onda especificados no método ocorre devido à presença de sistemas trienos e dienos conjugados respectivamente. Neste método, o óleo ou a gordura em questâo são dissolvidos em solvente apropriado e a extinção da solução é determinada nos comprimentos de onda especificados, usando como referência o solvente puro.

- Perfil de ácidos graxos: determinado por cromatografia gasosa, conforme metodologia de Hartmann e LAGO (1973). Por este método, os ésteres metílicos de ácidos graxos são separados, identificados e quantificados por cromatografia em fase gasosa. O método é aplicável para a determinação de ésteres metílicos de ácidos graxos, contendo de 4 a 24 átomos de carbono, obtidos a partir de ácidos graxos de óleos e gorduras. As condiçóes cromatográficas utilizadas foram: temperatura inicial da coluna igual a $40^{\circ} \mathrm{C}$ por cinco minutos, aumentada a uma taxa de $10^{\circ} \mathrm{C}$ minuto $^{-1}$ até a temperatura de $140{ }^{\circ} \mathrm{C}$, permanecendo 15 minutos, até temperatura final da coluna de $240{ }^{\circ} \mathrm{C}$ com aquecimento de $4{ }^{\circ} \mathrm{C}$ minuto ${ }^{-1}$, permanecendo por 30 minutos. $\mathrm{O}$ gás de arraste utilizado foi o nitrogênio ultrapuro com um fluxo de $1 \mathrm{~mL}$ minuto $^{-1}$. A temperatura do injetor e detector foi de $260^{\circ} \mathrm{C}$. A identificação dos diferentes ácidos graxos foi realizada por comparaçáa entre os tempos de retenção das amostras e dos padróes (CARdoso et al., 2010). 


\section{RESULTADOS E DISCUSSÃO}

$\mathrm{Na}$ polpa, o maior percentual de umidade foi verificado nas cultivares MGS GRAP084 (81,0\%), MGS GRAP556 (79,0\%), MGS MISS293 (80,6\%) e MGS ROP398 (78,9\%). No caroço, os maiores valores foram observados nas cultivares Arberquina (42,8\%), MGS ASC323 (42,9\%), MGS VIGLIONE (44,1\%), Empeltre (43,8\%), MGS MISS293 (43,5\%), Picual (43,8\%) e MGS ROP398 (42,9\%) (Tabela 1).

Em relação ao teor de lipídeos, as cultivares MGS ASC315 (63,9\%), Cerignola (62,9\%), MGS GRAP561 $(61,9 \%)$ e MGS GRAP575 (63,8\%) proporcionaram maiores porcentagens na polpa. No caroço, as cultivares MGS GRAP575 e MGS TAF390 tiveram maiores porcentagens, respectivamente, com 19,1\% e 18,6\%.

Esses resultados assemelham-se aos obtidos por Del Rio et al. (2005), em que esses autores observaram

Tabela 1. Composição centesimal para umidade e lipídeos de cultivares de oliveira. EPAMIG/UFLA, Maria da Fé (MG), 2011

\begin{tabular}{lrrrr} 
& \multicolumn{2}{c}{ Umidade (\%) } & \multicolumn{2}{c}{ Lipídeos (\%) } \\
\cline { 2 - 5 } Cultivar & Polpa & Caroço & Polpa & Caroço \\
\hline Alto D'Ouro & $57,7 \mathrm{f}$ & $31,0 \mathrm{~g}$ & $52,1 \mathrm{~d}$ & $6,7 \mathrm{e}$ \\
\hline Arbequina & $66,7 \mathrm{e}$ & $42,8 \mathrm{a}$ & $60,0 \mathrm{~b}$ & $13,6 \mathrm{c}$ \\
\hline Ascolano USA & $72,6 \mathrm{c}$ & $38,9 \mathrm{~d}$ & $57,9 \mathrm{c}$ & $8,5 \mathrm{e}$ \\
\hline Cerignola & $72,5 \mathrm{c}$ & $37,6 \mathrm{e}$ & $62,9 \mathrm{a}$ & $9,5 \mathrm{~d}$ \\
\hline Empeltre & $71,8 \mathrm{c}$ & $43,8 \mathrm{a}$ & $57,0 \mathrm{c}$ & $16,9 \mathrm{~b}$ \\
\hline Galega & $64,3 \mathrm{e}$ & $34,0 \mathrm{e}$ & $57,0 \mathrm{c}$ & $10,0 \mathrm{~d}$ \\
\hline JB1 & $66,5 \mathrm{e}$ & $32,1 \mathrm{~g}$ & $50,2 \mathrm{e}$ & $8,8 \mathrm{~d}$ \\
\hline MGS ASC315 & $72,3 \mathrm{c}$ & $36,7 \mathrm{e}$ & $63,9 \mathrm{a}$ & $7,6 \mathrm{e}$ \\
\hline MGS ASC322 & $69,2 \mathrm{~d}$ & $39,9 \mathrm{c}$ & $39,3 \mathrm{~g}$ & $13,9 \mathrm{c}$ \\
\hline MGS ASC323 & $71,1 \mathrm{c}$ & $42,9 \mathrm{a}$ & $57,0 \mathrm{c}$ & $13,3 \mathrm{c}$ \\
\hline MGS GRAP084 & $81,0 \mathrm{a}$ & $38,5 \mathrm{~d}$ & $52,5 \mathrm{~d}$ & $6,8 \mathrm{e}$ \\
\hline MGS GRAP541 & $67,4 \mathrm{~d}$ & $38,5 \mathrm{~d}$ & $57,6 \mathrm{c}$ & $7,3 \mathrm{e}$ \\
\hline MGS GRAP550 & $74,1 \mathrm{~b}$ & $41,5 \mathrm{~b}$ & $48,9 \mathrm{e}$ & $6,6 \mathrm{e}$ \\
\hline MGS GRAP553 & $71,5 \mathrm{c}$ & $40,0 \mathrm{c}$ & $59,4 \mathrm{~b}$ & $9,4 \mathrm{~d}$ \\
\hline MGS GRAP556 & $79,0 \mathrm{a}$ & $40,0 \mathrm{c}$ & $39,0 \mathrm{f}$ & $5,7 \mathrm{e}$ \\
\hline MGS GRAP561 & $65,3 \mathrm{e}$ & $36,4 \mathrm{e}$ & $61,9 \mathrm{a}$ & $14,9 \mathrm{c}$ \\
\hline MGS GRAP575 & $69,8 \mathrm{~d}$ & $39,8 \mathrm{c}$ & $63,8 \mathrm{a}$ & $19,1 \mathrm{a}$ \\
\hline MGS MANZ215 & $71,7 \mathrm{c}$ & $41,9 \mathrm{~b}$ & $59,1 \mathrm{~b}$ & $13,4 \mathrm{c}$ \\
\hline MGS MANZ234 & $73,6 \mathrm{~b}$ & $41,0 \mathrm{c}$ & $57,7 \mathrm{c}$ & $7,9 \mathrm{e}$ \\
\hline MGS MARIENSE & $74,9 \mathrm{~b}$ & $41,1 \mathrm{c}$ & $37,2 \mathrm{~g}$ & $10,2 \mathrm{~d}$ \\
\hline MGS MISS293 & $80,6 \mathrm{a}$ & $43,5 \mathrm{a}$ & $55,7 \mathrm{c}$ & $8,3 \mathrm{e}$ \\
\hline MGS NEBLINA & $69,4 \mathrm{~d}$ & $39,4 \mathrm{~d}$ & $52,6 \mathrm{~d}$ & $10,2 \mathrm{~d}$ \\
\hline MGS ROP398 & $78,9 \mathrm{a}$ & $42,9 \mathrm{a}$ & $37,8 \mathrm{~g}$ & $8,5 \mathrm{e}$ \\
\hline MGS SAL488 & $69,39 \mathrm{~d}$ & $39,42 \mathrm{~d}$ & $52,58 \mathrm{~d}$ & $10,23 \mathrm{~d}$ \\
\hline MGS SEVERO & $68,5 \mathrm{~d}$ & $41,7 \mathrm{~b}$ & $55,9 \mathrm{c}$ & $17,4 \mathrm{~b}$ \\
\hline MGS TAF390 & $68,0 \mathrm{~d}$ & $42,5 \mathrm{~b}$ & $62,3 \mathrm{c}$ & $18,6 \mathrm{a}$ \\
\hline MGS TAF391 & $71,5 \mathrm{c}$ & $41,3 \mathrm{~b}$ & $55,7 \mathrm{c}$ & $10,2 \mathrm{~d}$ \\
\hline MGS VIGLIONE & $69,9 \mathrm{~d}$ & $44,1 \mathrm{a}$ & $43,9 \mathrm{f}$ & $11,4 \mathrm{~d}$ \\
\hline MGS ZAL010 & $66,2 \mathrm{e}$ & $38,9 \mathrm{~d}$ & $49,6 \mathrm{e}$ & $6,1 \mathrm{e}$ \\
\hline Mission & $68,5 \mathrm{~d}$ & $33,3 \mathrm{f}$ & $49,5 \mathrm{e}$ & $7,9 \mathrm{e}$ \\
\hline Negroa & $68,6 \mathrm{~d}$ & $31,4 \mathrm{~g}$ & $52,7 \mathrm{~d}$ & $7,4 \mathrm{e}$ \\
\hline Cicual .V. (\%) & $72,5 \mathrm{c}$ & $43,8 \mathrm{a}$ & $57,1 \mathrm{c}$ & $9,1 \mathrm{~d}$ \\
\hline
\end{tabular}

Médias seguidas de mesma letra nas colunas nấo diferem entre si pelo teste de ScottKnott a 5\% de probabilidade. a variação de $32,2 \%$ a 40,1\% de lipídeos nas cultivares mantidas no Banco de Germplasma de Córdoba e 37,4\% a $54,3 \%$ de lipídeos nas cultivares mantidas no Banco de Germoplasma da Catalunha, ambos na Espanha.

Na polpa da cultivar Arbequina, observou-se a maior percentagem de proteína, 14,0\%. No caroço, as maiores porcentagens de proteínas foram constatadas nas cultivares Empeltre $(4,6 \%)$ e MGS GRAP556 (5,5\%). Para cinzas, os frutos da oliveira 'MGS ASC323' proporcionaram o maior resultado na polpa $(15,7 \%)$ e para a cultivar Picual, o maior resultado foi observado no caroço (4,9\%) (Tabela 2).

TANILGAN et al. (2007), ao caracterizarem cinco cultivares de oliveiras na regiáo da Anatólia, na Turquia, observaram teores de umidade que variaram de $36,8 \%$ a 59,2\%; lipídeos de $17,7 \%$ a $43,5 \%$; proteínas de $0,8 \%$ a $2,0 \%$ e cinzas de $0,6 \%$ a $1,2 \%$ nos frutos inteiros, resultados inferiores aos obtidos no presente trabalho, com exceção dos teores de umidades que se assemelharam.

Tabela 2. Composição centesimal para proteínas e cinzas de cultivares de oliveira. EPAMIG/UFLA, Maria da Fé (MG), 2011

\begin{tabular}{lrrrr} 
& \multicolumn{2}{c}{ Proteína (\%) } & \multicolumn{2}{c}{ Cinza (\%) } \\
\cline { 2 - 5 } Cultivar & Polpa & Caroço & Polpa & Caroço \\
\hline Alto D'Ouro & $8,3 \mathrm{~g}$ & $2,5 \mathrm{f}$ & $9,5 \mathrm{c}$ & $0,4 \mathrm{j}$ \\
\hline Arbequina & $14,0 \mathrm{a}$ & $3,7 \mathrm{c}$ & $7,2 \mathrm{f}$ & $0,3 \mathrm{j}$ \\
\hline Ascolano USA & $6,2 \mathrm{j}$ & $2,1 \mathrm{~h}$ & $9,7 \mathrm{c}$ & $2,0 \mathrm{e}$ \\
\hline Cerignola & $5,3 \mathrm{k}$ & $1,3 \mathrm{j}$ & $8,3 \mathrm{~d}$ & $0,9 \mathrm{~h}$ \\
\hline Empeltre & $10,3 \mathrm{~d}$ & $4,6 \mathrm{a}$ & $7,6 \mathrm{e}$ & $0,7 \mathrm{i}$ \\
\hline Galega & $9,3 \mathrm{f}$ & $4,3 \mathrm{~b}$ & $7,6 \mathrm{e}$ & $0,3 \mathrm{j}$ \\
\hline JB1 & $7,5 \mathrm{~h}$ & $3,2 \mathrm{e}$ & $6,2 \mathrm{~g}$ & $0,4 \mathrm{j}$ \\
\hline MGS ASC315 & $5,2 \mathrm{k}$ & $1,6 \mathrm{i}$ & $10,4 \mathrm{~b}$ & $1,4 \mathrm{f}$ \\
\hline MGS ASC322 & $5,5 \mathrm{k}$ & $2,5 \mathrm{~g}$ & $8,7 \mathrm{~d}$ & $1,4 \mathrm{f}$ \\
\hline MGS ASC323 & $5,0 \mathrm{I}$ & $2,0 \mathrm{~h}$ & $15,7 \mathrm{a}$ & $1,5 \mathrm{f}$ \\
\hline MGS GRAP084 & $9,9 \mathrm{e}$ & $2,4 \mathrm{~g}$ & $6,7 \mathrm{f}$ & $0,3 \mathrm{j}$ \\
\hline MGS GRAP541 & $6,8 \mathrm{i}$ & $2,2 \mathrm{~h}$ & $9,1 \mathrm{~d}$ & $0,8 \mathrm{i}$ \\
\hline MGS GRAP550 & $3,2 \mathrm{p}$ & $1,3 \mathrm{j}$ & $5,5 \mathrm{~h}$ & $0,3 \mathrm{j}$ \\
\hline MGS GRAP553 & $3,6 \mathrm{o}$ & $2,1 \mathrm{~h}$ & $6,2 \mathrm{~g}$ & $2,6 \mathrm{c}$ \\
\hline MGS GRAP556 & $3,0 \mathrm{p}$ & $5,5 \mathrm{a}$ & $6,0 \mathrm{f}$ & $3,0 \mathrm{~b}$ \\
\hline MGS GRAP561 & $4,6 \mathrm{~m}$ & $1,6 \mathrm{i}$ & $7,8 \mathrm{e}$ & $1,1 \mathrm{~g}$ \\
\hline MGS GRAP575 & $4,7 \mathrm{~m}$ & $2,8 \mathrm{f}$ & $8,4 \mathrm{~d}$ & $3,0 \mathrm{~b}$ \\
\hline MGS MANZ215 & $4,7 \mathrm{~m}$ & $1,4 \mathrm{j}$ & $6,6 \mathrm{f}$ & $1,5 \mathrm{f}$ \\
\hline MGS MANZ234 & $4,9 \mathrm{I}$ & $1,3 \mathrm{j}$ & $7,8 \mathrm{e}$ & $1,9 \mathrm{e}$ \\
\hline MGS MARIENSE & $5,3 \mathrm{k}$ & $3,4 \mathrm{~d}$ & $6,1 \mathrm{~g}$ & $0,6 \mathrm{i}$ \\
\hline MGS MISS293 & $3,7 \mathrm{o}$ & $1,3 \mathrm{j}$ & $9,6 \mathrm{c}$ & $1,1 \mathrm{~g}$ \\
\hline MGS NEBLINA & $3,4 \mathrm{p}$ & $1,5 \mathrm{j}$ & $7,0 \mathrm{f}$ & $1,9 \mathrm{e}$ \\
\hline MGS ROP398 & $6,7 \mathrm{i}$ & $2,6 \mathrm{f}$ & $9,3 \mathrm{c}$ & $0,3 \mathrm{j}$ \\
\hline MGS SAL488 & $4,10 \mathrm{n}$ & $2,55 \mathrm{f}$ & $7,24 \mathrm{f}$ & $1,62 \mathrm{f}$ \\
\hline MGS SEVERO & $4,4 \mathrm{n}$ & $2,1 \mathrm{~h}$ & $3,3 \mathrm{i}$ & $1,6 \mathrm{f}$ \\
\hline MGS TAF390 & $5,3 \mathrm{k}$ & $2,4 \mathrm{~g}$ & $7,7 \mathrm{e}$ & $1,6 \mathrm{f}$ \\
\hline MGS TAF391 & $4,1 \mathrm{n}$ & $1,9 \mathrm{~h}$ & $9,2 \mathrm{c}$ & $2,2 \mathrm{~d}$ \\
\hline MGS VIGLIONE & $11,5 \mathrm{~b}$ & $3,7 \mathrm{c}$ & $8,7 \mathrm{~d}$ & $0,5 \mathrm{j}$ \\
\hline MGS ZAL010 & $10,7 \mathrm{c}$ & $3,5 \mathrm{~d}$ & $7,8 \mathrm{e}$ & $0,4 \mathrm{j}$ \\
\hline Mission & $8,7 \mathrm{~g}$ & $3,9 \mathrm{c}$ & $9,3 \mathrm{c}$ & $0,3 \mathrm{j}$ \\
\hline C.V. (\%) & $1,6 \mathrm{~g}$ & $2,4 \mathrm{~g}$ & $5,5 \mathrm{~h}$ & $0,4 \mathrm{j}$ \\
\hline
\end{tabular}

Médias seguidas de mesma letra nas colunas não diferem entre si pelo teste de ScottKnott a 5\% de probabilidade. 
O índice de acidez em ácido oleico variou de 0,2\% (Tabela 3). Este índice influencia diretamente na acidez final do azeite, e quanto menor esta acidez, melhor a qualidade. Um azeite classificado como extravirgem, possui menos de 0,8\% de acidez, sendo considerado de altíssima qualidade e o mais saudável e completo entre todos os azeites, além de ser responsável pelos efeitos benéficos à saúde humana (ANVISA, 2009).

O índice de acidez pode ser influenciado por fatores como maturação, estocagem, ação enzimática, qualidade da azeitona, sistema de obtenção do azeite (extração mecânica e/ou por solvente), grau de refinação e pureza (Регхото et al., 1998). Entretanto, em algumas cultivares o azeite estava com valores acima do permitido pela ANVISA, que é de $0,8 \%$, tais como: MGS ASC322 $(1,0 \%)$, MGS ASC323 (1,1\%), Cerignola (1,4\%), MGS ZAL010 (0,9\%) e MGS GRAP556 (0,9\%), podendo estar relacionado com as condiçôes climáticas no dia em que foram realizadas as extraçóes e maior tempo de armazenamento dos frutos antes do processamento.

No índice de peróxido, observaram-se resultados que variaram de 5,840 $\mathrm{mmol} \mathrm{kg}^{-1}$ (cv. MGS MISS293) a 19,460 mmol $\mathrm{kg}^{-1}$ (cv. MGS GRAP575) (Tabela 3). O índice de peróxido pode ser afetado pelos condicionantes pós-colheita. Além disso, o processo de extração pode influenciar no índice de peróxido, pela oxidação inicial, a rancificação do azeite ou a deterioração que pode ocorrer nos antioxidantes naturais, como os tocoferóis e os polifenóis (CARDOSO et al., 2010).

Quanto ao índice de iodo, este variou de 70,620 (cv. MGS GRAP553) a 87,220 $\mathrm{g} \mathrm{I}_{2} \mathrm{~kg}^{-1}$ (cv. MGS ZAL010) estando todas as amostras analisadas dentro do padrão brasileiro de óleos e gorduras para o azeite de oliva (75 a $90 \mathrm{~g} \mathrm{I}_{2} \mathrm{~kg}^{-1}$ ) (ANVISA, 2005) (Tabela 3). Este índice refere-se à quantidade de insaturação que o material lipídico possui, sendo benéfico à saúde o consumo deste tipo de

Tabela 3. Composição química dos azeites extraídos das cultivares de oliveira. EPAMIG/UFLA, Maria da Fé (MG), 20111

\begin{tabular}{|c|c|c|c|c|c|c|}
\hline Cultivares & $\begin{array}{c}\text { AAO } \\
(\%)\end{array}$ & $\begin{array}{c}\text { IP } \\
\left(\mathrm{mmol}_{\mathrm{c}} \mathbf{k g}^{-1}\right)\end{array}$ & $\begin{array}{c}\text { II } \\
\left(\mathrm{g} \mathrm{I}_{2} \mathrm{~kg}^{-1}\right)\end{array}$ & $\begin{array}{c}\text { IRA } \\
\left(+20^{\circ} \mathrm{C}\right)\end{array}$ & $\begin{array}{c}\text { AUV } \\
(270 \mathrm{~nm})\end{array}$ & $\begin{array}{c}\text { AUV } \\
(232 \mathrm{~nm})\end{array}$ \\
\hline Alto D’Ouro & 0,2 & 10,50 & 75,57 & 1,470 & 0,045 & 1,26 \\
\hline Arbequina & 0,2 & 6,93 & 81,45 & 1,470 & 0,160 & 1,49 \\
\hline Ascolano USA & 0,4 & 8,62 & 81,01 & 1,469 & 0,185 & 2,46 \\
\hline Cerignola & 1,4 & 16,45 & 80,68 & 1,469 & 0,221 & 3,26 \\
\hline Empeltre & 0,2 & 11,00 & 83,25 & 1,470 & 0,060 & 2,14 \\
\hline Galega & 0,2 & 10,80 & 74,96 & 1,470 & 0,110 & 2,40 \\
\hline JB1 & 0,4 & 6,64 & 75,71 & 1,470 & 0,120 & 2,00 \\
\hline MGS ASC 315 & 0,7 & 10,30 & 78,10 & 1,470 & 0,142 & 1,84 \\
\hline MGS ASC 322 & 1,0 & 13,54 & 79,05 & 1,469 & 0,240 & 2,11 \\
\hline MGS ASC 323 & 1,1 & 13,24 & 77,40 & 1,469 & 0,200 & 0,24 \\
\hline MGS GRAP084 & 0,2 & 9,31 & 76,06 & 1,469 & 0,140 & 1,39 \\
\hline MGS GRAP541 & 0,2 & 7,92 & 82,84 & 1,470 & 0,090 & 1,55 \\
\hline MGS GRAP550 & 0,2 & 9,61 & 79,16 & 1,470 & 0,163 & 2,13 \\
\hline MGS GRAP553 & 0,3 & 12,88 & 70,62 & 1,469 & 0,253 & 3,02 \\
\hline MGS GRAP556 & 0,9 & 10,10 & 80,91 & 1,469 & 0,216 & 3,41 \\
\hline MGS GRAP561 & 0,2 & 11,69 & 84,57 & 1,470 & 0,090 & 1,16 \\
\hline MGS GRAP575 & 0,3 & 19,46 & 78,27 & 1,470 & 0,198 & 2,31 \\
\hline MGS MANZ215 & 0,2 & 13,27 & 82,27 & 1,470 & 0,190 & 2,41 \\
\hline MGS MANZ234 & 0,5 & 11,09 & 82,70 & 1,469 & 0,226 & 2,62 \\
\hline MGS MARIENSE & 0,2 & 10,10 & 75,70 & 1,470 & 0,080 & 1,62 \\
\hline MGS MISS293 & 0,2 & 5,84 & 80,08 & 1,469 & 0,142 & 1,84 \\
\hline MGS NEBLINA & 0,5 & 9,21 & 81,20 & 1,469 & 0,170 & 1,88 \\
\hline MGS ROP398 & 0,5 & 10,95 & 83,69 & 1,470 & 0,070 & 1,46 \\
\hline MGS SAL488 & 0,2 & 11,79 & 75,03 & 1,470 & 0,090 & 1,40 \\
\hline MGS SEVERO & 0,4 & 11,47 & 86,19 & 1,469 & 0,120 & 1,31 \\
\hline MGS TAF391 & 0,5 & 11,39 & 81,30 & 1,470 & 0,123 & 1,81 \\
\hline MGS TAF390 & 0,5 & 8,32 & 82,24 & 1,469 & 0,060 & 1,25 \\
\hline MGS VIGLIONE & 0,4 & 10,50 & 80,77 & 1,469 & 0,170 & 1,53 \\
\hline MGS ZAL010 & 0,9 & 9,21 & 87,22 & 1,469 & 0,305 & 2,60 \\
\hline Mission & 0,4 & 10,70 & 76,65 & 1,470 & 0,100 & 1,69 \\
\hline Negroa & 0,4 & 9,01 & 71,45 & 1,470 & 0,152 & 1,87 \\
\hline Picual & 0,4 & 13,13 & 76,07 & 1,470 & 0,205 & 2,20 \\
\hline Referências ${ }^{(1)}$ & Máx. 0,8 & Máx. 20,0 & $75,0-90,0$ & $1,4677-1,4705$ & Máx. 0,22 & Máx. 2,5 \\
\hline
\end{tabular}

AAO: Acidez em ácido oleico; IP: Índice de peróxido; II: Índice de iodo; IRA: Índice de refraçấo absoluto; AUV: Absorbância em ultra violeta. ( $\left.{ }^{1}\right)$ Resoluçâo n. 270 da ANVISA (ANVISA, 2005). 
gordura, pois auxilia na prevenção de doenças cardiovasculares, uma vez que tem a aptidão de reduzir os níveis de triglicerídeos e o colesterol sanguíneo, elevando o HDL (conhecido como colesterol bom) e diminuindo o nível do LDL (conhecido como colesterol ruim). Além disso, o índice de iodo é uma medida que possibilita a identificação de adulteraçóes em diversos tipos de óleos.

Para o índice de refração absoluto a $20{ }^{\circ} \mathrm{C}$, houve pequena variação entre as cultivares, ou seja, de 1,469 a 1,470 (Tabela 3). A absorbância em ultravioleta a $270 \mathrm{~nm}$ variou de 0,045 para a cultivar Alto D'Ouro a 0,305 na cultivar MGS ZAL010 e a absorbância em ultravioleta a $232 \mathrm{~nm}$, variou de 0,240 (cv. MGS ASC323) a 3,410 (cv. MGS GRAP556) (Tabela 3). Embora na maioria das cultivares houve valores adequados, as cultivares Cerignola, MGS ZAL010, MGS GRAP553 e MGS MAN234 tiveram valores acima do permitido para ambas as variáveis. Além disso, a cultivar MGS ASC322 proporcionou valores acima para $270 \mathrm{~nm}$ e a cultivar MGS GRAP556 para $232 \mathrm{~nm}$.
No presente estudo, reforça-se a idéia que a não conformidade deste índice para algumas amostras esteja relacionado com o método de extração, ainda experimental, e condiçôes ambientais no momento da extração das amostras.

Com relação ao perfil dos ácidos graxos, foram encontrados seis ácidos graxos nas amostras dos azeites analisados: ácido palmítico, ácido palmitoleico, ácido esteárico, ácido oleico, ácido linoleico e ácido linolênico, sendo o ácido oleico o de maiores quantidades, entre $71,4 \%$ (cv. MGS Neblina) e 88,5\% (cv. MGS GRAP084) (Tabela 4). AgANCHich et al. (2008) observaram 75,3\% em um experimento com a cultivar Picholine marocaine, enquanto Gomes-Rico et al. (2008), verificaram valores entre $55,9 \%$ e $79,5 \%$ de ácido oleico, para as cultivares Cornicabra e Morisca.

O ácido oleico é considerado o principal ácido graxo presente no azeite e apresenta ação benéfica à saúde. Estudos realizados por Menezes et al. (2005) comprovaram a sua eficácia no tratamento de câncer de mama. Pesquisas

Tabela 4. Composição do perfil dos principais ácidos graxos observados nas amostras dos azeites. EPAMIG/UFLA, Maria da Fé (MG), 2011

\begin{tabular}{|c|c|c|c|c|c|c|}
\hline \multirow[b]{2}{*}{ Cultivares } & \multicolumn{6}{|c|}{ Ácidos graxos (\%) } \\
\hline & $\begin{array}{c}\text { Ácido } \\
\text { palmítico }\end{array}$ & $\begin{array}{c}\text { Ácido } \\
\text { palmitoleico }\end{array}$ & $\begin{array}{c}\text { Ácido } \\
\text { esteárico }\end{array}$ & $\begin{array}{l}\text { Ácido } \\
\text { oleico }\end{array}$ & Ácido linoleico & $\begin{array}{l}\text { Ácido } \\
\text { linolênico }\end{array}$ \\
\hline Alto D’Ouro & 12,2 & 0,9 & 1,7 & 75,9 & 8,7 & 0,60 \\
\hline Arbequina & 14,1 & 1,8 & 0,1 & 73,5 & 9,6 & 0,00 \\
\hline Ascolano USA & 11,8 & 0,8 & 1,0 & 78,1 & 7,6 & 0,00 \\
\hline Cerignola & 11,9 & 1,0 & 1,6 & 78,5 & 7,1 & 0,00 \\
\hline Cornicabra & 15,8 & 2,5 & 0,0 & 75,8 & 5,9 & 0,00 \\
\hline Empeltre & 13,8 & 1,4 & 0,0 & 73,8 & 10,6 & 0,00 \\
\hline Galega & 15,6 & 3,2 & 1,2 & 74,7 & 3,8 & 0,45 \\
\hline JB1 & 15,8 & 3,0 & 0,0 & 77,7 & 3,5 & 0,00 \\
\hline MGS ASC315 & 12,6 & 1,1 & 1,5 & 77,1 & 7,3 & 0,00 \\
\hline MGS ASC322 & 9,8 & 0,0 & 0,0 & 87,6 & 2,2 & 0,00 \\
\hline MGS ASC 323 & 12,2 & 1,4 & 0,0 & 83,2 & 2,7 & 0,00 \\
\hline MGS GRAP084 & 11,6 & 0,0 & 0,0 & 88,5 & 0,0 & 0,00 \\
\hline MGS GRAP541 & 6,8 & 0,3 & 1,3 & 87,1 & 4,4 & 0,00 \\
\hline MGS GRAP550 & 12,1 & 0,0 & 0,0 & 83,7 & 4,1 & 0,00 \\
\hline MGS GRAP553 & 17,9 & 0,0 & 0,0 & 82,1 & 0,0 & 0,00 \\
\hline MGS GRAP556 & 10,8 & 1,1 & 0,9 & 80,0 & 6,4 & 0,00 \\
\hline MGS GRAP561 & 8,6 & 0,2 & 0,0 & 84,1 & 7,0 & 0,00 \\
\hline MGS GRAP575 & 13,3 & 0,0 & 1,0 & 79,5 & 5,7 & 0,00 \\
\hline MGS MANZ215 & 9,7 & 0,0 & 0,0 & 85,0 & 5,3 & 0,00 \\
\hline MGS MANZ234 & 12,2 & 0,9 & 1,7 & 75,9 & 8,7 & 0,60 \\
\hline MGS MARIENSE & 15,8 & 2,2 & 1,7 & 75,2 & 4,5 & 0,50 \\
\hline MGS MISS293 & 12,6 & 1,1 & 1,5 & 77,1 & 7,3 & 0,00 \\
\hline MGS NEBLINA & 14,9 & 1,5 & 1,6 & 71,4 & 10,7 & 0,00 \\
\hline MGS ROP398 & 13,8 & 1,4 & 0,0 & 74,1 & 10,8 & 0,00 \\
\hline MGS SEVERO & 10,4 & 0,4 & 0,0 & 76,9 & 9,9 & 1,00 \\
\hline MGS TAF390 & 9,3 & 0,4 & 0,0 & 84,9 & 5,1 & 0,00 \\
\hline MGS TAF391 & 17,2 & 3,1 & 0,0 & 79,7 & 0,0 & 0,00 \\
\hline MGS VIGLIONE & 8,0 & 0,4 & 2,0 & 85,3 & 3,0 & 0,70 \\
\hline MGS ZAL010 & 1,1 & 10,6 & 2,1 & 81,6 & 4,0 & 0,00 \\
\hline Mission & 14,0 & 2,1 & 1,2 & 78,0 & 3,3 & 0,50 \\
\hline Negroa & 17,2 & 0,0 & 0,0 & 78,8 & 4,1 & 0,00 \\
\hline Picual & 14,5 & 1,2 & 1,5 & 77,1 & 5,0 & 0,00 \\
\hline Referências (1) & $7,5-20,0$ & $0,3-3,5$ & $0,5-5,0$ & $55,0-83,0$ & $3,5-21,0$ & $0,0-1,5$ \\
\hline
\end{tabular}

(1) Codex Stan 33 - rev. 2003 (Codex Alimentarium Comission, 2003). 
realizadas por Kris-Etherton et al. (1999) e LARSEN et al. (1999) enfatizaram o papel do azeite na proteçáo cardiovascular, sendo muitos dos efeitos saudáveis atribuídos ao alto conteúdo de ácido oleico presente no azeite.

Com a avaliação de diversos outros parâmetros, como: composição de esteróis, conteúdo de eritrodiol e uvaol, estigmastadieno, ECN42, Delta-K composiçẫo de triacilgliceróis, entre outros, como citados por Oliveira et al. (2010a), é possível avaliar com mais segurança a identidade e qualidade dos azeites. Desta forma, é de fundamental importância o aprimoramento técnico dos laboratórios brasileiros no controle de óleos vegetais, pois uma avaliação mais refinada poderá indicar índices ainda mais elevados de adulteraçáo nos azeites comercializados e futuramente fabricados no Brasil.

\section{CONCLUSÃO}

As cultivares MGS ASC315, Cerignola, MGS GRAP561 e MGS GRAP575 possuem maiores valores de lipídeos. Todas as cultivares avaliadas tiveram índices de acidez em ácido oleico, peróxidos e refração absoluta de acordo com a resolução da ANVISA, podendo ser inicialmente classificados como azeite de oliva extravirgem ou virgem.

De modo geral, todos os ácidos graxos presentes nos azeites possuem valores satisfatórios, destacando a cultivar MGS GRAP084, com maior concentração de ácido oleico, principal ácido responsável pelos benefícios à saúde.

\section{AGRADECIMENTOS}

À Fundação de Amparo a Pesquisa do Estado de Minas Gerais - FAPEMIG (CAG APQ 02721/09), pelo apoio financeiro na execução desse trabalho e à Coordenação de Aperfeiçoamento de Pessoal de Nível Superior (CAPES), pela concessão de bolsa de mestrado ao primeiro autor.

\section{REFERÊNCIAS}

AGANCHICH, B.; ANTARI A.E.L.; WAHBI, S.; TAHI, H.; WAKRIM, R.; SERRAJ, R. Fruit and oil quality of mature olive trees under partial rootzone drying in field conditions. Grasas y Aceites, v.59, p.225-233, 2008.

AGÊNCIA NACIONAL DE VIGILÂNCIA SANITÁRIA ANVISA. Resolução no 270, de 22 de setembro de 2005. Aprova o Regulamento Técnico para Óleos Vegetais, Gorduras Vegetais e Creme Vegetal. 2005. Disponível em: <http://www.azeiteonline.com.br/wpcontent/uploads/2011/04/anvisa-resolucao-rdc270-de-22-09-2005. pdf $>$. Acesso em: 10/5/2011.

AGÊNCIA NACIONAL DE VIGILÂNCIA SANITÁRIA ANVISA. Regulamento Técnico para Fixaçáo de Identidade e Qualidade de Óleos e Gorduras vegetais. 2009. Disponível em: <http://www4.anvisa.gov.br/base/visadoc/CP/CP\%5B8994-10\%5D.pdf >. Acesso em: 4/8/2011.
ASSOCIATION OF OFFICIAL ANALYTICAL CHEMISTRY AOAC. Official methods of analysis of the Association of Official Analytical Chemistry. 17.ed. Washington, 2005. 1410p.

AUED-PIMENTEL, S.; TAKEMOTO, E.; KUMAGAI, E.E.; CANO, C.B. Determinaçáo da diferença entre o valor real e o teórico do triglicerídeo ECN 42 para a detecção de adulteração em azeites de oliva comercializados no Brasil. Revista Química Nova, v.31, p.31-34, 2008.

CARDOSO, L.G.V.; BARCELOS, M.F.P.; OLIVEIRA, A.F.; PEREIRA, J.A.R.; ABREU, W.C.; PIMENTEL, F.A.; CARDOSO, M.G.; PEREIRA, M.C.A. Características físico-químicas e perfil de ácidos graxos de azeites obtidos de diferentes variedades de oliveiras introduzidas no Sul de Minas Gerais - Brasil. Semina Ciências Agrárias, v.31, p.127-136, 2010.

CODEX ALIMENTARIUS COMISSION. Standard for olive oils and olive pomace oils. Codex Stan 33. Italy: FAO/WHO, 1981, revisão 2003. 8p. Disponível em: <http://www.codexalimentarius. net/web/more_info.jsp?id_sta=88>. Acesso em: 9/5/ 2010.

DEL RÍO, C.; CABALLERO, J.M.; GARCÍA-FERNADÉZ, M.D.; TOUS, J.; ROMERO, A. Rendimiento graso de la aceituna. In: RALLO, L.; BARRANCO, D.; CABALLERO, J. M.; DEL RÍO, C.; MARTÍN, A.; TOUS, J.; TRUJILLO, I. (Ed.). Variedades de olivo en España. Sevilla: Consejería de Agricultura y Pesca de la Junta de Andalucía; Madrid: Ministerio de Agricultura, Pesca y Alimentación/Mundi-Prensa, 2005. cap.13, p.349-356.

FERREIRA, D.F. Sisvar: a computer statistical analysis system. Ciência e Agrotecnologia, v.35, p.1039-1042, 2011.

GÓMEZ-RICO, A.; SALVADOR, M.D.; FREGAPANE, G. Virgin olive oil and olive fruit minor constituents as affected by irrigation management based on SWP and TDF as compared to ETc in medium-density young olive orchards (Olea europaea L. cv. Cornicabra and Morisca). Food Research International, v.42, p.1067-1076, 2009.

HARTMAN, L.; LAGO, R.C.A. A rapid preparation of fatty acid methyl esters from lipids. Laboratorie Practice, v.22, p.475-476, 1973.

INSTITUTO ADOLFO LUTZ - IAL. Normas analíticas do Instituto Adolfo Lutz: métodos químicos e físicos para análise de alimentos. 3.ed. São Paulo, 2005. v.1, 533p.

KRIS-ETHERTON, P.M.; PEARSON, T.A.; WAN, Y.; HARGROVE, R.L.; MORIATRTY, K.; FISHELL, V.; ETHERTON, T.D. High-monounsaturated fatty acid diets lower both plasma cholesterol and triacylglycerol concentrations. American Journal of Clinical Nutrition, v.70, p.1009-1015, 1999.

LARSEN, L.F.; JESPERSEN, J.; MARCKMANN, P. Are olive oil diets antithrombotic? Diets enriched with olive, rapeseed, or sunflower oil affect postprandial factor VII differently. American Journal of Clinical Nutrition, v.70, p.976-982, 1999.

MINISTÉRIO DA AGRICULTURA, PECUÁRIA E ABASTECIMENTO - MAPA. Instrução Normativa n.1, de 30 de janeiro de 2012. 2012. Disponível em: <http:// www.azeiteonline.com.br/wp-content/uploads/2012/02/ INSTRU\%C3\% 87\%C3\%83O-NORMATIVA-N\%C2\%BA1-DE-30-DE-JANEIRO-DE-2012_MAPA.pdf $>$. Acesso em: $2 / 2 / 2012$ 
MENEZES, J.A.; VELLON, L.; COLOMER, R.; LUPU, R. Oleic acid, the main monounsaturated fatty acid of olive oil, suppresses Her-2/neu (erbB-2) expression and synergistically enhances the growth inhibitory effects of trastuzumab (Herceptin $^{\mathrm{TM}}$ ) in breast cancer cells with Her-2/ neu oncogene amplification. Annals of Oncology, v.3, p.359-371, 2005.

OLIVEIRA, A.F.; VIEIRA NETO, J.; GONÇALVES, E.D.; VILLA, F.; SILVA, L.F.O. Parâmetros físico-químicos dos primeiros azeites de oliva brasileiros extraídos em Maria da Fé, Minas Gerais. Scientia Agraria, v.11, p.255-261, 2010a.

OLIVEIRA, M.C.; VIEIRA NETO, J.; OLIVEIRA, R.S.; PIO, R.; OLIVEIRA, N.C.; RAMOS, J.D. Enraizamento de estacas de duas cultivares de oliveira submetidas à aplicação de diferentes fertilizantes. Bragantia, v.69, p.99-103, 2010b.

OLIVEIRA, M.C.; RAMOS, J.D.; PIO, R.; SANTOS, V.A.; SILVA, F.O.R. Enraizamento de estacas em cultivares de oliveiras promissores para a Serra da Mantiqueira. Ceres, v.59, p.147-150, 2012 a.

OLIVEIRA, M.C.; RAMOS, J.D.; PIO, R.; CARDOSO, M.G. Características fenológicas e físicas e perfil de ácidos graxos em oliveiras no sul de Minas Gerais. Pesquisa Agropecuária Brasileira, v.47, p.30-35, 2012b.

PEIXOTO, E.R.M.; SANTANA, D.M.N.; ABRANTES, S. Avaliação dos índices de identidade e qualidade do azeite de oliva:
Proposta para atualização da legislação brasileira. Boletim da Sociedade Brasileira de Ciência e Tecnologia de Alimentos, v.18, p.363-470, 1998.

PIO, R.; BASTOS, D.C.; BERTI, A.J.; FILHO, J.A.S.; MOURÃO FILHO, F.A.A.; ENTELMANN, F.A.; ALVES, A.S.R.; NETO, J.E.B. Enraizamento de diferentes tipos de estacas de oliveira (Olea europaea L.) utilizando ácido indolbutírico. Ciência e Agrotecnologia, v.29, p.562-567, 2005.

SÁNCHEZ, S.; PACHECO, R.; RUBIA, M.D.; SÁNCHEZ, A.; PEREIRA, M.G. Aplicación de distintos microtalcos natulares, como coadyuvantes tecnólógicos, en los procesos de extracción de aceites de oliva. Jaen: TEC 65, Expoliva, 2005. 4p.

SUÁREZ, J.M.M; ARANDA, M.; MENDOZA, J.; REY, A.L. Informe sobre utilización del analizador de rendimentos "Abencor". Grasas y Azeite, v.26, p.379-385, 1975.

TANILGAN, K.; ÖZCANB, M.M.; ÜNVERB, A. Physical and chemical characteristics of five Turkish olive (Olea europea L.) varieties and their oils. Grasas y Aceites, v.58, p.142-147, 2007.

VIEIRA NETO, J.; OLIVEIRA, A.F; OLIVEIRA, N.C.; DUARTE, H.S.S.; GONÇALVES, E.D. Aspectos técnicos da cultura da oliveira. Belo Horizonte: EPAMIG, 2008. 56p. (EPAMIG, Boletim Técnico 88) 